\title{
Response: Sarcopenia Is Significantly Associated with Presence and Severity of Nonalcoholic Fatty Liver Disease ( $\mathrm{J}$ Obes Metab Syndr 2019;28:129-38)
}

Goh Eun Chung, Ji Won Yoon*

Department of Internal Medicine, Seoul National University Hospital Healthcare System Gangnam Center, Seoul, Korea
Received May 19, 2020

Reviewed May 25, 2020

Accepted June 5, 2020

${ }^{*}$ Corresponding author

Ji Won Yoon

https://orcid.org/0000-0001-9003-0614

Department of Internal Medicine,

Seoul National University Hospital

Healthcare System Gangnam Center,

152 Teheran-ro, Gangnam-gu,

Seoul 06236, Korea

Tel: +82-2-2112-5677

Fax: +82-2-2112-5794

E-mail: jwyoonmd@gmail.com
Recently, we reported the association between sarcopenia and nonalcoholic fatty liver disease (NAFLD) in Journal of Obesity \& Metabolic Syndrome. ${ }^{1}$ In our study, younger age seemed to strongly impact the association between sarcopenia and NAFLD. However, this finding is not consistent, as shown in the findings by Kim et al. ${ }^{2}$ that older adults ( $\geq 45$ years) had a stronger association between sarcopenia and NAFLD.

The mechanism through which age is an effect modifier is not clear, but analyzing the clinical characteristics according to age, as suggested in the letter, would be helpful in estimating the components. Thus, we compared several body compositions and metabolic and inflammatory parameters according to age group.

In all subjects, with or without sarcopenia, waist circumference and visceral adipose tissue area increased while skeletal muscle mass decreased with age (data not shown). Similarly, blood pressure and blood glucose level increased with age. On the other hand, triglycerides, high-density lipoprotein (HDL)-cholesterol, and alanine aminotransferase levels were not significantly different among age groups. Also, high-sensitivity C-reactive protein (hs-CRP) level as an inflammatory marker was not significantly different according to age group (data not shown).

However, in subjects with sarcopenia, younger subjects were more likely to be male and had higher body mass index, waist circumference, and skeletal muscle mass (Table 1). They also showed higher uric acid, triglyceride, and total and low-density lipoprotein (LDL)-cholesterol level and lower HDL-cholesterol level. Again, hs-CRP level showed no difference (Table 1). Male sex, obesity/ abdominal obesity, and worse metabolic profiles shown in blood tests can be related to insulin resistance, an important risk factor for NAFLD. Even though sex and visceral adiposity were adjusted in the subgroup analysis of our previous study, the residual effect of insulin resistance might have influenced the association between sarcopenia and NALFD. In our study population, most young subjects with sarcopenia had sarcopenic obesity. Only two people in the tertile 1 (19-49 years) age group were non-obese and sarcopenic. Therefore, we were not able to compare metabolic profiles between young sarcopenic subjects with or without obesity.

A previous study by Srikanthan et al. ${ }^{3}$ reported that sarcopenia was more strongly associated with insulin resistance in a younger group ( $<60$ years). In that study, not only sarcopenic obesity, but 
Table 1. Clinical characteristics of subjects with sarcopenia according to age

\begin{tabular}{|c|c|c|c|c|c|}
\hline Variable & $\mathrm{T} 1(19-49 \mathrm{yr}, \mathrm{n}=82)$ & T2 (50-57 yr, n=65) & $\mathrm{T} 3(58-87 \mathrm{yr}, \mathrm{n}=168)$ & Total $(n=315)$ & $P$ \\
\hline Age (yr) & $42.1 \pm 4.8^{*,+}$ & $53.0 \pm 2.3^{\ddagger}$ & $65.9 \pm 6.0$ & $57.1 \pm 11.4$ & $<0.001$ \\
\hline Male (n, \%) & $69(84.1)$ & $48(73.8)$ & $113(67.3)$ & $230(73)$ & $0.018^{*}$ \\
\hline Systolic blood pressure (mmHg) & $123.0 \pm 13.1$ & $122.2 \pm 14.1$ & $125.4 \pm 14.7$ & $124.1 \pm 14.2$ & 0.228 \\
\hline Diastolic blood pressure (mmHg) & $0.8 \pm 10.7^{\dagger}$ & $79.4 \pm 10.8$ & $76.5 \pm 10.6$ & $78.2 \pm 10.8$ & 0.007 \\
\hline Waist circumference $(\mathrm{cm})$ & $99.5 \pm 9.0^{*, t}$ & $95.3 \pm 9.1$ & $94.7 \pm 8.7$ & $96.1 \pm 9.0$ & $<0.001$ \\
\hline Visceral adipose tissue $\left(\mathrm{cm}^{2}\right)$ & $171.8 \pm 52.5$ & $186.3 \pm 56.2$ & $180.2 \pm 60.6$ & $179.3 \pm 57.7$ & 0.322 \\
\hline Body mass index $\left(\mathrm{kg} / \mathrm{m}^{2}\right)$ & $29.8 \pm 3.9^{*, t}$ & $28.3 \pm 3.5^{\ddagger}$ & $26.7 \pm 3.1$ & $27.8 \pm 3.6$ & $<0.001$ \\
\hline Appendicular skeletal muscle (kg) & $22.8 \pm 4.1^{*,+}$ & $20.1 \pm 4.9^{\ddagger}$ & $18.2 \pm 4.5$ & $19.8 \pm 4.9$ & $<0.001$ \\
\hline ASM/body weight (\%) & $26.9 \pm 2.6^{\dagger}$ & $26.1 \pm 3.4$ & $25.9 \pm 3.1$ & $26.2 \pm 3.1$ & 0.044 \\
\hline Fasting glucose (mg/dL) & $108.0 \pm 38.5$ & $103.9 \pm 20.6$ & $106.5 \pm 22.8$ & $106.3 \pm 27.4$ & 0.661 \\
\hline Total cholesterol (mg/dL) & $05.3 \pm 37.0^{\dagger}$ & $199.8 \pm 42.0$ & $190.8 \pm 35.4$ & $196.5 \pm 37.7$ & 0.013 \\
\hline Triglycerides (mg/dL) & $50.0 \pm 59.2^{\dagger}$ & $132.5 \pm 67.0$ & $116.1 \pm 58.7$ & $128.6 \pm 62.2$ & $<0.001$ \\
\hline HDL-cholesterol (mg/dL) & $46.2 \pm 7.4^{\dagger}$ & $47.4 \pm 8.8^{\ddagger}$ & $50.4 \pm 11.0$ & $48.7 \pm 9.9$ & 0.004 \\
\hline LDL-cholesterol (mg/dL) & $37.3 \pm 34.7^{\dagger}$ & $130.9 \pm 36.0^{\ddagger}$ & $120.9 \pm 30.7$ & $127.4 \pm 33.6$ & 0.001 \\
\hline Alanine aminotransferase (IU/mL) & $45.3 \pm 28.5^{*, t}$ & $34.8 \pm 20.7$ & $31.7 \pm 20.4$ & $35.9 \pm 23.5$ & $<0.001$ \\
\hline Uric acid (mg/dL) & $7.1 \pm 1.5^{*, t}$ & $6.3 \pm 1.4^{\ddagger}$ & $5.7 \pm 1.6$ & $6.2 \pm 1.6$ & $<0.001$ \\
\hline hs-CRP (mg/dL) & $0.2 \pm 0.4$ & $0.1 \pm 0.2$ & $0.2 \pm 0.4$ & $0.2 \pm 0.3$ & 0.586 \\
\hline
\end{tabular}

Values are presented as mean \pm standard deviation or number (\%). One-way analysis of variance with post-hoc least significant difference for continuous variables. Chi-square test for categorical variable.

${ }^{*} P<0.05$ between $\mathrm{T} 1$ and $\mathrm{T} 2 ;{ }^{\dagger} P<0.05$ between $\mathrm{T} 1$ and $\mathrm{T} 3 ;{ }^{\ddagger} P<0.05$ between $\mathrm{T} 2$ and $\mathrm{T} 3$.

T1, 1st tertile; T2, 2nd tertile; T3, 3rd tertile; ASM, appendicular skeletal muscle; HDL, high-density lipoprotein; LDL, low-density lipoprotein; hs-CRP, high-sensitivity C-reactive protein.

also sarcopenia without obesity was significantly associated with insulin resistance. However, data from Korea National Health and Nutrition Examination Survey 2009-2010 showed no age-related effect modification between sarcopenia and insulin resistance. ${ }^{4}$ In a study reporting decrease in skeletal muscle mass as an independent risk factor for incident NAFLD, the subjects in a younger age subgroup ( $<45$ years) did not show such an association. ${ }^{5}$ However, in non-obese subjects, decreased muscle mass over time was a risk factor for NAFLD even in a younger age group. ${ }^{5}$ As such, findings of the association between sarcopenia and insulin resistance or NALFD vary depending on the study. The discrepancy between study results could be due to differences in study design and population characteristics.

In our study, an increase in insulin resistance at a younger age could be explained in part by obesity/abdominal obesity in this group. Unfortunately, detailed analysis of the association between sarcopenia with or without obesity and NAFLD was difficult to perform using the data. Differences in etiology of sarcopenia, changes in muscle fiber type distribution, and intramyocellular lipid accumulation have been suggested as possible mechanisms of age-related differences in the association between sarcopenia and insulin resistance. ${ }^{3}$ Further studies are needed to determine whether there is an effect modification by age and whether these factors cause differences in insulin resistance.

The effect of chronic inflammation in the association between sarcopenia and NAFLD did not seem to be major. However, chronic inflammation is regarded as an important mediator of insulin resistance, ${ }^{6}$ so future studies should evaluate it as a potential factor for mediating the association between sarcopenia and NAFLD. We are grateful for the opportunity to look deeper into our data based on this response letter.

\section{CONFLICTS OF INTEREST}

The authors declare no conflict of interest.

\section{AUTHOR CONTRIBUTIONS}

Study concept and design: all authors; acquisition of data: all authors; analysis and interpretation of data: all authors; drafting of the manuscript: JWY; critical revision of the manuscript: GEC; statistical analysis: all authors. 


\section{REFERENCES}

1. Chung GE, Kim MJ, Yim JY, Kim JS, Yoon JW. Sarcopenia is significantly associated with presence and severity of nonalcoholic fatty liver disease. J Obes Metab Syndr 2019;28:129-38.

2. Kim HY, Kim CW, Park CH, Choi JY, Han K, Merchant AT, et al. Low skeletal muscle mass is associated with non-alcoholic fatty liver disease in Korean adults: the Fifth Korea National Health and Nutrition Examination Survey. Hepatobiliary Pancreat Dis Int 2016;15:39-47.

3. Srikanthan P, Hevener AL, Karlamangla AS. Sarcopenia exacerbates obesity-associated insulin resistance and dysglycemia: findings from the National Health and Nutrition Examination
Survey III. PLoS One 2010;5:e10805.

4. Moon SS. Low skeletal muscle mass is associated with insulin resistance, diabetes, and metabolic syndrome in the Korean population: the Korea National Health and Nutrition Examination Survey (KNHANES) 2009-2010. Endocr J 2014;61: 61-70.

5. Lee MJ, Kim EH, Bae SJ, Kim GA, Park SW, Choe J, et al. Age-related decrease in skeletal muscle mass is an independent risk factor for incident nonalcoholic fatty liver disease: a 10 year retrospective cohort study. Gut Liver 2019;13:67-76.

6. Theuma P, Fonseca VA. Inflammation, insulin resistance, and atherosclerosis. Metab Syndr Relat Disord 2004;2:105-13. 